\title{
Identification of the factors affecting co-localization precision for quantitative multicolor localization microscopy
}

\author{
Paolo Annibale ${ }^{1 *}$, Marco Scarselli ${ }^{1,2}$, Mattia Greco ${ }^{1}$ and Aleksandra Radenovic ${ }^{\text {* }}$
}

\begin{abstract}
This work explores the potential of multi-color Photoactivated Localization Microscopy (PALM) imaging to probe sub-diffraction limit interactions between proteins with spectrally separated labels. Using a PALM setup built around a commercial microscope axially stabilized to $\mathrm{nm}$-level, we determined the ultimate registration accuracy that could be achieved $(10 \mathrm{~nm})$ and compared the performance of three different pairs of fluorescent proteins that can be used in dual color PALM. Fusion constructs were cloned and imaged either in vitro or at the cell plasma membrane, allowing to identify a current limit to co-localization precision of approximately 30-40 nm. We identified the better performing pair and present a concluding perspective application to a co-clustering study.
\end{abstract}

Keywords: Super-resolution, Photoactivated localization microscopy, Axial stability, Registration precision, Endocytosis

\section{Background}

One of the basic concepts in fluorescence microscopy is the use of photons from spectrally separated fluorophores to distinguish differently labeled species within a cell. This concept has been successfully applied since decades in the cell biology field. The detection of the multiple emitted wavelengths can be performed either sequentially, using the same detector, or simultaneously, using two or more independent detectors (channels). The information obtained from the relative position or overlap of the fluorescent emission in the pixels of two or more detection channels can be used, for example, to draw conclusions about the relative affinity or interaction between labeled species within a cell (Manders et al. 1992; Manders et al. 1993).

Reference markers, such as uniform, multiply stained microspheres, are typically used to align the two different images of the same scene, in a process that is well known as registration of the two images (Additional file 1: Figure S1). The tolerances required by diffraction limited imaging are in the order of one Airy minimum (0.61 $\lambda$ ), within the wavelength interval $\lambda=250 \mathrm{~nm}-450 \mathrm{~nm}$ in the visible range.

\footnotetext{
* Correspondence: paolo.annibale@epfl.ch; aleksandra.radenovic@epfl.ch 'Laboratory of Nanoscale Biology, Institute of Bioengineering, School of Engineering, EPFL, Lausanne 1015, Switzerland

Full list of author information is available at the end of the article
}

However, super-resolution microscopy, in particular the approach based on switching single molecules stochastically in space followed by their localization (Betzig et al. 2006; Hess et al. 2006; Rust et al. 2006), introduces four major challenges to this picture. First, the pixel size of superresolution images is below $10 \mathrm{~nm}$, leading to a drastic decrease in the tolerance of the registration process. Second, the stochastic photoactivation and localization process implies that in most of the cases neighboring fluorophores will be activated in widely separated instants of time, making the control of mechanical drifts and the overall stability of the setup of paramount importance. Third, the photoactivation (switching) of the probes determines special constraints on the pairs of photoactivatable Fluorescent Proteins (PAFPs) that can be used (Shroff et al. 2007) that add to the requirement of spectral distinguishability. Finally, the extended duration of these experiments challenges traditional methods of sample fixation to keep individual fluorophores immobilized at the nanoscale for the time required to buildup a PALM image.

The first multi-color super-resolution imaging papers appeared in 2007, and were: Bates et al. dualcolor STORM (Bates et al. 2007) investigation using the fluorescent label Cy2-Alexa647 and Cy3-Alexa-647; and Shroff et al. dualcolor PALM paper (Shroff et al. 2007), introducing the 
pairs of photoactivatable fluorescent proteins tdEosDronpa/PSCFP2. The former work observed the relationship between microtubules and clathrin coated pits, whereas the latter focused on proteins belonging to the adhesion complex machinery; in both cases a supercolocalization was not observed; in the case of the adhesion complex study, in fact, it was concluded that the two species do not interact since they do not display sub-diffraction limit colocalization. In neither of these papers the registration protocol used nor its accuracy were explicitly addressed. A 2008 paper (Huang et al. 2008) by the Zhuang group investigated the relationship between mitochondria and the microtubule network by means of dual-color STORM. In the same year the Jakobs group (Andresen et al. 2008) proposed a group of new PA-FPs for use in dual-color studies, as well as an alternative approach to multicolor measurements based on the use of spectrally closely spaced probes to be assigned to a specific color based on the ratio of the photons detected for each emitter in each of the spectral channels.

This approach was further extended by Testa et al. in 2010 (Testa et al. 2010), to triple channel detection using Alexa dyes, whereas in the pure PALM field the sequential imaging approach based on the use of mEos2 and Dronpa was applied to the study of dendritic spines (Frost et al. 2010). Approaches based on the use of new, red PA-FPs allowing for simultaneous imaging using PAGFP as a green partner, were reported in 2009 and 2010 by the Lippincott-Schwartz group (Subach et al. 2009; Subach et al. 2010), in studies focusing on the assembly and clustering of plasma membrane proteins. The year 2010 was marked also by a key publication discussing the ultimate limits to registration and co-localization of spectrally separated emitters in CCD based measurements (Pertsinidis et al. 2010). Active stabilization of the imaging optics allows for sub-nanometer registration accuracy, with the caveat that emitters need to fall within the same camera pixel for such a precision to hold. In 2011 another application of the mEos2-PSCFP2 pair (Yao et al. 2011), as well as two works using mEos2-Dronpa combination were published (Hsu and Baumgart 2011) (Lehmann et al. 2011). A successful multi-color strategy was also demonstrated using dSTORM suitable dyes (Baddeley et al. 2011) or a combination of PALM/STORM approaches, as in the recent work by Hess and coworkers (Wilmes et al. 2012), leading to three color live cell super-resolution imaging. Since the original development of PALM/STORM in 2006, a large number of photoswitchable fluorescent proteins or labeling strategies with organic dyes are now available (Patterson et al. 2010). This work focuses only on the use of PAFPs for two color super-resolution studies. The motivation for this approach is that it requires the minimum amount of staining and sample manipulation, aside from the DNA transfection, and therefore strategies to optimize its performance should be actively investigated. In addition, it should not be neglected that the one label-one protein condition offered by PALM is the prerequisite that will be able to ensure the most quantitative outcome among all the super-resolution methods.

Therefore we first direct our efforts to establish a protocol that can be followed in any multi color localization microscopy experiment in order to maximize the colocalization precision. We combine a previously published registration approach (Churchman et al. 2005) to the implementation of an axial stabilization system that can be readily set-up in any commercial or laboratory microscope.

We then present the original use of PAFPs fusion pairs to evaluate the performance of pairs of photo activatable fluorescent proteins, exploiting the fixed stoichiometry of these constructs to focus on the quantitation possibilities offered by each of the pairs. We limited our study to three pairs, but more combinations are and will become available as soon as new PAFPs are developed, and we therefore believe that this strategy will be instrumental to determine the best combination of fluorophores for multicolor PALM depending on the desired application.

After identifying the better performing pair we present a perspective application to a co-clustering study in a biological system, discussing the important role of chemical fixation as a concluding remark.

\section{Methods \\ Plasmids}

The mEos2 gene is cloned into the plasmid pRSETA (Addgene plasmid 20341, original material provided by Loren Looger HHMI). The small peptide SrcN15 represents the N-terminal 15 amino acids of the protein Src, it is myristoylated, and was demonstrated to be associated to the non-raft domains of the plasma membrane (Chichili and Rodgers 2007). We purchased the fluorophore PSCFP2 from the company Evrogen as ps-CFP2-N (117997, Moscow, Russia) for mammalian expression.

The two DNA constructs SrcN15-mEos2 and SrcN15PSCFP2 were synthesized and cloned in the mammalian expression vector pJ603 by the DNA 2.0 company (94025 CA, USA).

For the constructs $\beta 2$-PSCFP2 we subcloned the $\beta 2$ gene (cDNA, Missouri ST cDNA Resource center) at the $\mathrm{N}$-terminus of PSCFP2 amplifying by PCR the $\beta 2$ sequence with the primers CTTAAGCTTGGTACCACC ATGTAC (sense) and CGCGGGCCCCCAGCAGTG AGTCATT (antisense) in order to create the EcoRI and ApaI restriction sites.

PAMCherry1-clathrin light chain and PSCFP2-clathrin light chain were a kind gift of Michael W. Davidson (National High Magnetic Field Laboratory, Florida State University, USA). The PAMCherry1 construct was a 
kind gift of Prof. Jennifer Lippincott-Schwartz (NIH, Bethesda, USA).

The MN1 Dronpa plasmid (MBL Amalgaam) was opened using the restriction sites HindIII and NotI.

The mEos2-Dronpa construct was generated in the mammalian expression vector pJ603 from the company DNA 2.0 (Menlo Park, USA). It was subcloned in the pRSETA bacterial expression vector for protein expression in BL21(DE3) pLysS competent cells using a NotI and ApaI restriction sites. mEos2 and Dronpa were separated by a four alanines linker.

The construct coding for S15-PSCFP2-mEos2 was purchased from DNA 2.0. The sequence was subcloned in the pRSET1 plasmid upon removal of SRCN15 to enhance bacterial expression using the primers: AAGG ATCGATGGGGATCCATGGTGATGAGCAAGGGCGC CGAGCTG (sense) and GGCGGCGGTCACGAACTCG AGGTAGATCATGTGATCGCGCTT (antisense) allowing for restriction using Xhol and NcoI.

The S15-PSCFP2-PAMCherry1 sequence is spaced respectively by four and three alanines. The sequence was expressed in the mammalian pJ603 and subcloned in the bacterial expression vector pRSETA using the restriction sites NotI and ApaI.

\section{Protein purification and in vitro sample preparation}

All pRSETA plasmids were transformed into the Bacterial strain BL21(DE3) pLysS (Stratagene, La Jolla, CA, USA). Cultures were grown overnight in Medium containing $100 \mathrm{mg} / \mathrm{mL}$ ampicillin and $25 \mathrm{mg} / \mathrm{mL}$ chloramphenicol to a 0.4-0.5 optical density and then induced with $1 \mathrm{mM}$ IPTG at $37^{\circ} \mathrm{C}$ for five hours.

The cells were harvested from a $500 \mathrm{ml}$ culture by centrifugation and resuspended in eight $\mathrm{ml}$ of lysis buffer $\left(500 \mathrm{mM} \mathrm{NaCl}, 50 \mathrm{mM} \mathrm{NaH} \mathrm{PO}_{4}, \mathrm{pH}\right.$ 8). Then, eight $\mathrm{mg}$ of lysozyme was added and the reaction was incubated 30 minutes in ice. The solution was sonicated on ice five times with a sonicator equipped with a microtip using six $10 \mathrm{~s}$ bursts at high intensity with a $10 \mathrm{~s}$ cooling period between each burst. The lysate was centrifuged at $3000 \times \mathrm{g}$ for 15 minutes to pellet cellular debris. The supernatant was transferred to a fresh tube and added to an equilibrated Invitrogen Ni-NTA purification Column. Binding was allowed for one hour with gentle agitation to keep the resin suspended in the lysate solution. The resin was allowed to settle by centrifugation $(800 \times \mathrm{g})$ and the supernatant was carefully aspirated. The column was washed with $8 \mathrm{ml}$ of Native Wash Buffer with imidazole at low concentration (500 mM NaCl, $50 \mathrm{mM} \mathrm{NaH} \mathrm{PO}_{4}, \mathrm{pH} 8$ and $20 \mathrm{mM}$ imidazole). This last step was repeated three more times.

The column was clamped in a vertical position and the cap was removed from the lower end in order to elute the protein with $12 \mathrm{ml}$ native elution buffer $(500 \mathrm{mM} \mathrm{NaCl}$,
$50 \mathrm{mM}$ NaH2PO4, pH 8 and $250 \mathrm{mM}$ imidazole). After purification, the proteins were dialyzed in Slide-A-Lyzer cassettes with 10000 MWCO (Pierce Chemical Company, Rockford, IL) using PBS and then concentrated.

\section{In-vitro sample preparation}

Single molecule fluorescent proteins samples were obtained by mixing the aqueous solution containing the proteins to Acrylamide and crosslinking solution and vortexing. Gel (PAGE) was obtained by diluting in water a stock solution 30\% Acrylamide (Sigma) with 1.6\% Methylenebisacrylamide (Sigma) to a final Acrylamide concentration of $15 \%$. Polymerization was obtained, after a few cycles of degassing and nitrogen flow, by adding Ammonium Persulfate Solution and TEMED and mixing. $20 \mu \mathrm{L}$ of the polymerizing solution were immediately sandwiched between two $25.4 \mathrm{~mm}$ no. 1.5 clean circular glass coverslips. Fluorescence imaging was performed after $1 \mathrm{~h}$, and within at most a few hours, at room temperature.

\section{Cell cultures, transfection and sample fixation}

HeLa cells were grown in DMEM (without phenol red) supplemented with $10 \%$ fetal bovine serum, $100 \mathrm{mg} / \mathrm{ml}$ streptomycin, and 100 units $/ \mathrm{ml}$ penicillin at $37^{\circ} \mathrm{C}$ with $5 \% \mathrm{CO}_{2}$. The cells were transfected using the NeonTM Transfection System (Invitrogen) following the manufacturer's instructions. The electroporation was performed for a 6 well dish (500,000-1,000,000 cells/well) using 0.5-2 $\mu \mathrm{g}$ of DNA for each sample following the manufacturer's parameters (Voltage 1,005; 2 Pulses with a width of $35 \mathrm{~ms}$ ). Experiments were performed after 16-24 h after transfection. For the internalization of $\beta 2$-mEos2, isoprotenerol $10 \mu \mathrm{M}$ was used for $10 \mathrm{~min}$. The day of the experiment, after extensive washing with PBS, cells were fixed incubating with $4 \%$ paraformaldehyde and $0.2 \%$ glutaraldehyde at $37^{\circ} \mathrm{C}$ for $30 \mathrm{~min}$ in PHEM (60 mM Pipes, $25 \mathrm{mM}$ HEPES, $10 \mathrm{mM}$ EGTA, and $2 \mathrm{mM} \mathrm{MgCl}$, $\mathrm{pH}$ 6.9) and washed 5X with PHEM. The coverslips, $25.4 \mathrm{~mm}$ diameter, no. 1.5 thickness, were cleaned in a solution obtained by mixing $125 \mathrm{ml}$ of water with $25 \mathrm{ml}$ of ammonium hydroxide and $25 \mathrm{ml}$ of $\mathrm{H}_{2} \mathrm{O}_{2} 30 \%$. A further step based on repeated rinsing with MilliQ water, immersion in spectroscopic grade methanol, nitrogen blowing and a passage under the flame was performed.

\section{Equipment and settings}

The excitation setup, built on an independent optical platform, consists of three laser-diode lines, two for excitation (488 nm Coherent Sapphire 488, $50 \mathrm{~mW}$ ad $561 \mathrm{~nm}$ Spectra-Physics Excelsior, $150 \mathrm{~mW}$ ) and one for photo-activation (405 $\mathrm{nm}$ Coherent Cube, $50 \mathrm{~mW}$ ). The excitation light is amplitude modulated and gated through an Acousto-Optical Polychromatic Tunable Filter AOM 
(A.A. Optoelectronics), which allows an on-off switching of each line with a rise time of $1.5 \mathrm{~ms}$. Both the excitation and the activation beams are expanded (10-20X) and the activation beam is spatial filtered (pinhole size $10 \mathrm{um}$ ). The filtered and expanded activation beam is combined to the excitation beam-path by a Semrock R405-Di01 dichroic Mirror. The combined laser beams are focused to the back focal plane (BFP) of a 1.45 NA $100 \mathrm{X}$ (or 60 $\mathrm{X})$ Olympus Total Internal Reflection Fluorescence (TIR) objective by a $250 \mathrm{~mm}$ focal length, $50 \mathrm{~mm}$ diameter, achromatic doublet lens placed on a $x-y-z$ micrometric translation stage. TIR at the sample arises as the focused laser beam is translated away from the optical axis in the back focal plane of the objective, resulting in a controllable exit angle of the beam with respect to the optical axis. In our setup the beam angle is accurately controlled by the translation of the $250 \mathrm{~mm}$ achromatic doublet. A $9.5 \mathrm{~mm}$ thick Fused Silica Laser Grade window (SQW-2037-UV Melles Griot) placed on a rotating goniometer before the lens is used to translate the collimated beam and maintain the excitation spot in the center of the field of view as the doublet lens is translated.

The microscope is based on the structure of an inverted Olympus model IX 71, with a camera side-port and an excitation port at the rear of the frame.

Excitation and emission are separated by a multiband dichroic mirror (Semrock FF493/574-Di01), and fluorescent emission is observed through a Photometrics DV2 beamsplitter containing a Chroma T565lpxr dichroic and two Semrock bandpass filters (FF593/40 and FF520/35). The long and short wavelength channels are imaged on the same CCD chip, projected each on one half of the entire chip.

Single molecule fluorescence images are detected by an Andor Technology iXon + DU-897E electron multiplying CCD camera, after a $2 \mathrm{X}$ further magnification, yielding a pixel size of $80 \mathrm{~nm}$ (or $133 \mathrm{~nm}$ ). Custom developed LabView software controls the PALM acquisition sequence, triggering the $\mathrm{CCD}$ detector at each excitation cycle. The CCD detector in turn, has the possibility to trigger the AOM, to avoid any stray light on the CCD chip and therefore bleed-through during readout. Data is spooled to hard drive for post-processing.

The $561 \mathrm{~nm}$ excitation power, as measured in epifluorescence mode after the objective, resulted in power densities at the sample in the range of $250-1000 \mathrm{~W} / \mathrm{cm}^{2}$. Datasets were recorded by reaching to both ends of this power range. For both mEos2 and PS-CFP we used activation powers at $405 \mathrm{~nm}$ in the in the range of $10-100 \mathrm{~mW} / \mathrm{cm}^{2}$.

The sample is mounted within a steek custom coverslip holder, attached to a three-axes nanopositioning stage (LPS-100, Mad City Labs) via a low thermal expansion coefficient aluminium holder.
The reflected beam of a TIR laser line can be exploited as a sensor of the relative objective to sample distance. Figure 1a displays a schematic of the optical setup devised to drive a feedback loop in order to keep the relative sample to objective distance constant. The laser line from a $785 \mathrm{~nm}$ pigtailed diode laser (Thorlabs, LPS785-FC) is collimated by a $12.5 \mathrm{~mm}$ lens and focused on BFP of the objective after reflection on a half mirror attached to the microscope frame near the rear excitation port and placed in close proximity to the path of the excitation beams. The diode is placed in a heat sink (Thorlabs LM9LP) and is driven by a power supply (Thorlabs, EK2000). The collimated beam is then focused on the BFP far enough from the optical axis to undergo total internal reflection at the sample-coverslip interface. The reflected laser line is steered back by two half mirrors on a quadrant photo diode (QPD Pacific Silicon QP50-6SD2), where the reflected spot moves along one direction proportionally to the separation between the objective lens and the coverslip. The difference signal from the two halves of the photodiode is then supplied to the NI USB 6229 acquisition card, and provides the input for the software PID controlling the nano-positioning stage $\mathrm{z}$-axis by serial communication.

The software PID controller, a part of the custom PALM control software that we developed, (LabView, National Instruments, Austin, Texas, USA), receives as an input the voltage value from the Top-Bottom output of the QPD. The PID controller compares this value to the set-point, corresponding to the signal measured when the sample is in focus, and acts on the $\mathrm{z}$-axis of the nano-positioning stage to maintain their difference (error) minimal. Before each measurement it is necessary to calibrate the $\mathrm{nm} / \mathrm{mV}$ ratio by moving up and down in steps the nano positioning stage.

\section{Results and discussion}

Protocol for image registration in dual color localization microscopy

\section{Determination of mapping function}

Generally speaking, image registration is the process of aligning two or more images of the same scene. The first of the challenges of two-color super-resolution microscopy is to achieve routinely and reliably a good registration precision of the order of, or smaller than, the localization precision of the probes. We therefore directed our efforts to establish a registration protocol that could be readily implemented after any PALM measurement in any commercial or laboratory setup. We adapted and elaborated the concept, introduced by Churchman and Spudich in 2005 (Churchman et al. 2005), based on the use of fluorescent fiducial markers to achieve the registration of two images detected on the two halves of a CCD chip after separating the sample's fluorescence through a dichroic beamsplitter. 

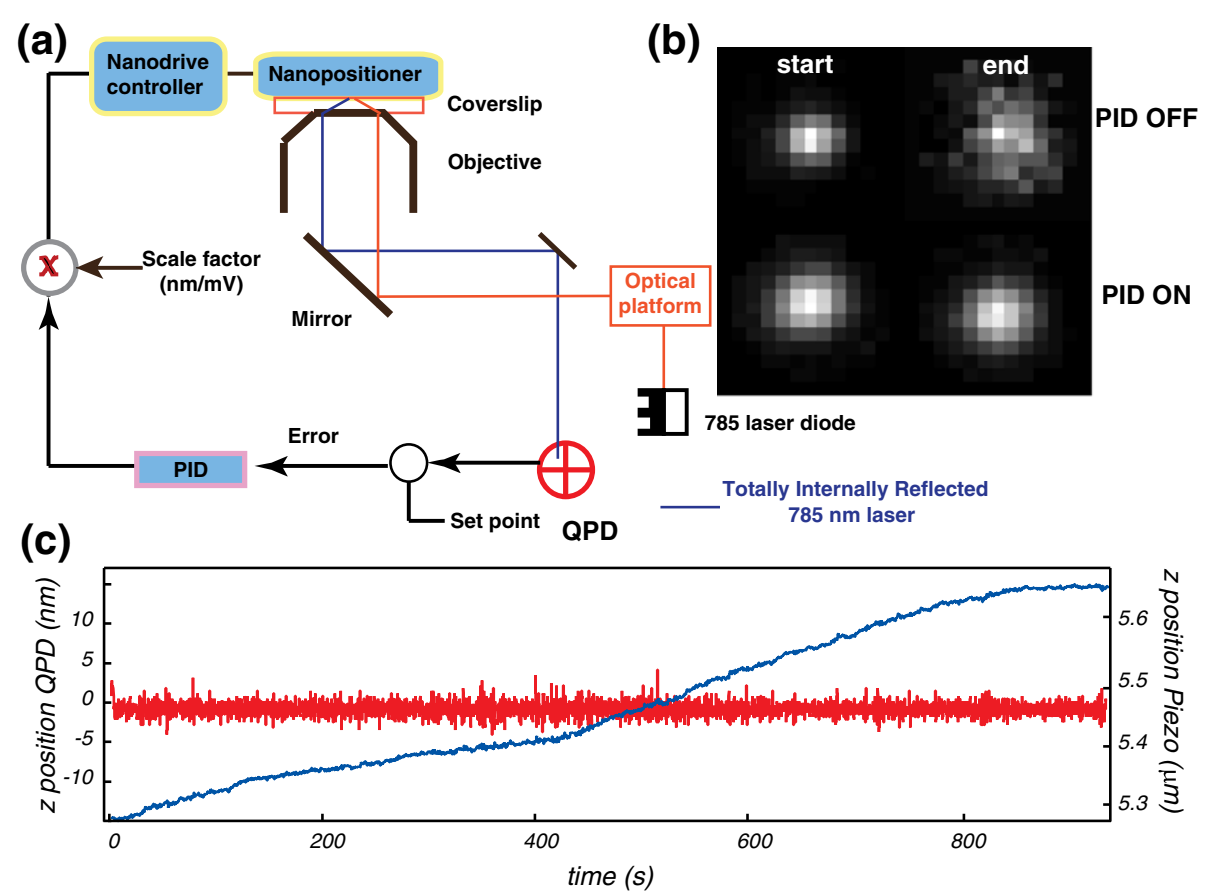

Figure 1 Registration protocol. (a) Schematics of the optical setup obtained to achieve axial stabilization. A near-infrared laser line from a pigtailed diode (Thorlabs, Newton, New Jersey) is collimated and focused off-axis in the back-focal plane of the high NA TIRF objective used for imaging. The totally internally reflected laser-line (blue) is then steered on a quadrant photodiode QP50-6SD2 (Pacific Silicon, West Lake Village, California). (b) The net output of this setup is to maintain the relative distance between the objective and the sample fixed (i.e. the focal position), avoiding blurring of the PSF over time. (c) A trace measured from the QPD over time, showing the feed-back signal (in red) and the zdisplacement of the nano-positioning stage to counter the axial offset. The system is accurate over extended periods of time within $5 \mathrm{~nm}$ peak-to-peak.

We used the $\mathrm{x}, \mathrm{y}, \mathrm{z}$ sample piezo-stage (See Equipment and Settings) to scan an isolated fluorescent bead in a grid, as displayed in Additional file 1: Figure S1. The fluorescent bead (Tetraspeck, Invitrogen) is a $100 \mathrm{~nm}$ diameter microsphere, stained with four different fluorescent dyes simultaneously: blue $(365 / 430 \mathrm{~nm})$, green (505/ $515 \mathrm{~nm})$, orange $(560 / 580 \mathrm{~nm})$ and dark red $(660 / 680 \mathrm{~nm})$. It is excited simultaneously by both the $488 \mathrm{~nm}$ and a $561 \mathrm{~nm}$ lasers, and imaged on the EM-CCD camera with an integration time of $0.5 \mathrm{~s}$. Each second the piezo moves the bead to the next position and another image is taken.

The typical step size used to generate the grids for registering a PALM acquisition was $21 \times 21$ steps spaced by either $250 \mathrm{~nm}$ or $500 \mathrm{~nm}$, hence covering 20 to $100 \mu \mathrm{m}^{2}$.

The position of the peaks in each image and in each channel is localized based on a maximum likelihood Gaussian fitting (Mortensen et al. 2010). Up to $m$ user defined peaks per frame can be fit, for each of the $N$ frames used to generate the complete grid. In each frame the peaks are identified based on their brightness. The pair of $\left(\mathrm{x}_{\mathrm{i}}, \mathrm{y}_{\mathrm{i}}\right)$ coordinates from each fitted pair of corresponding peaks is then used to calculate the mapping function required for image registration.

The function used to map the localized red channel peaks onto the green channel is the local weighted mean algorithm introduced by Goshtasby (Goshtasby 1988) in 1988, and first applied by Churchman et al. in the framework of single molecule experiments. The algorithm relies on the use of control points evenly spread throughout the field of view to locally evaluate polynomial functions to map each point of image 1 into the corresponding control point in image 2 . Weights are introduced in order to decrease the influence of polynomials the further away from a given control point. The calculation of this mapping function, given the coordinates of a set of control points in the two imaging channels, is performed using the Matlab (The MathWorks) library cp2tform. The registration between the two channels of our acquisition is calculated off line, after that the actual super-resolution imaging sequence is performed and that the registration grid has been acquired (as detailed in Additional file 1: Figure S2).

\section{Figure of merit of mapping function}

To quantify the accuracy of the registration, we first used the Fiducial Registration Error (FRE) (Churchman et al. 2005), defined here (along one axis) as $F R E_{x}=$ $\frac{1}{N} \sum_{i}\left(x_{g}^{i}-f\left(x_{r}^{i}, y_{r}^{i}\right)\right)$, where $f$ is the function mapping the points in the red channel onto the green channel, and Additional file 1: Figure S1, shows that the average 
FRE decreases as a function of the marker localization precision, reaching a plateau below $4 \mathrm{~nm}$ when the marker localization precision is better than one $\mathrm{nm}$. A very similar approach in the context of super-resolution measurements was detailed also by Bates et al. (Bates et al. 2012) using fiducial markers randomly arranged in the sample, however this approach yielded a poorer FRE, and no information was provided to monitor the registration accuracy over time, as it is detailed in the next two paragraphs.

\section{nm-level axial stabilization}

As the channel registration holds at one point in time and at one image plane, throughout the relatively long PALM image acquisition, the registration precision is challenged by the interplay of a variation of the sample axial position and a mechanical displacement of the imaging optics of one channel with respect to the other one. Furthermore, it was demonstrated (Engelhardt et al. 2011) that, in the presence of molecular dipoles that are not free to rotate, axial offsets can result into sizable lateral offsets. This phenomenon can result in up to $2.8 \mathrm{~nm}$ lateral offset for $10 \mathrm{~nm}$ axial displacement. To alleviate these two problems an axial stabilization system was put in place. The axial stabilized system is based on a feedback system using the total internal reflection of a near-infrared laser line, a concept originally proposed by Huang et al. (Huang et al. 2008) and Pertsinidis et al. (Pertsinidis et al. 2010), and it is described in the Equipment and Settings section. As Figure 1 (b) displays, the sensitivity of this setup allows not only to avoid defocus and macroscopic blurring of the Point Spread Function (PSF) of individual emitters, but also to maintain the axial position of the sample stable to the nm-level with a $5 \mathrm{~nm}$ accuracy for extended periods of time.

\section{Empirical determination of registration accuracy}

By measuring a time-lapse movie of the position of a bright fluorescent bead in both imaging channels (Buckers et al. 2011; Nugent-Glandorf and Perkins 2004), we can now estimate how the stability of the experimental setup affects the mapping function over time. The spontaneous lateral $x-y$ drift allows the bead to explore an extended area of the field of view, in between the registration points, as displayed in Figure 2a. The scanned fluorescent bead is localized to better than $2 \mathrm{~nm}$ (Figure $2 \mathrm{~b}$ ) in both imaging channels and its position in the red channel before and after the application of the mapping function is displayed. The remaining $\mathrm{x}$ and $\mathrm{y}$ offsets after mapping are displayed in the scatter plot of Figure 2c. Notably, many points scatter beyond the expected error given by the combination of the localization precisions and the FRE (inner circle in Figure $2 \mathrm{c}, \mathrm{r}=4.5 \mathrm{~nm}$ ). The average value of the registration offset in our setup was $6.7 \mathrm{~nm}$, with a standard deviation of $3.7 \mathrm{~nm}$, therefore $50 \%$ larger than the expected value. This implies that only $84 \%$ of the events are registered better than $10 \mathrm{~nm}$, and all of the points are within a registration precision of $\sim 18 \mathrm{~nm}$. We regard these figures as the empirical registration accuracy of the setup, and we suggest than in any circumstance this empirical value (eREG) should be calculated and used instead of the FRE.

The reason why the offset between the position of the bead in the two channels does not fall below the expected sum of the FRE and the particles' localization precision for all of the points is quite complex to address. We will focus our discussion here to the fact that the relative drift in between the imaging channels may play a role by increasing the residual offset. Additional file 1: Figure S3 displays the $\mathrm{x}-\mathrm{y}$ drift of a fiducial marker in an actual dual color measurement: the fiducial drift follows a slightly different path when measured in each channel, and the relative drift, that should be constant, fluctuates approximately $10 \mathrm{~nm}$ (as shown in lower inset in Additional file 1: Figure S3). It was demonstrated that this relative drift can corrected using an active stabilization of the imaging path, but only in a proof-of-principle single molecule experiment (Pertsinidis et al. 2010).

\section{Summary of the protocol for maximizing the registration precision}

As a summary of this section we recommend to perform channel registration using a closely $(<500 \mathrm{~nm})$ spaced grid of control points, to be generated with a precise $x-y$ stage. The registration can be performed either prior to or after the localization experiment, depending on the fluorophores used (e.g. dark to bright or bright to another color).

Maintaining the focal plane constant throughout the acquisition and the registration process, will maximize imaging and registration accuracy. In the methods section, we present an inexpensive method to implement an axial stabilization system on any commercial or laboratory setup.

The empirical registration accuracy of the setup over time can be established by mapping the position of a bright marker imaged in both channels over time.

\section{Choice of a pair of photoactivatable fluorescent proteins}

Next, we directed our efforts to identify best performing fluorescent probe pairs for dual color super-resolution microscopy experiments. An extensive and thorough characterization for multicolor STORM dyes has been recently performed by Dempsey et al. (Dempsey et al. 2011). A similar study was not yet reported for PAFPs suitable for multicolor PALM studies.

A large number of possible fluorescent probe pairs were reported to date as candidates to perform dual color super-resolution microscopy experiments. We restricted 
(a)

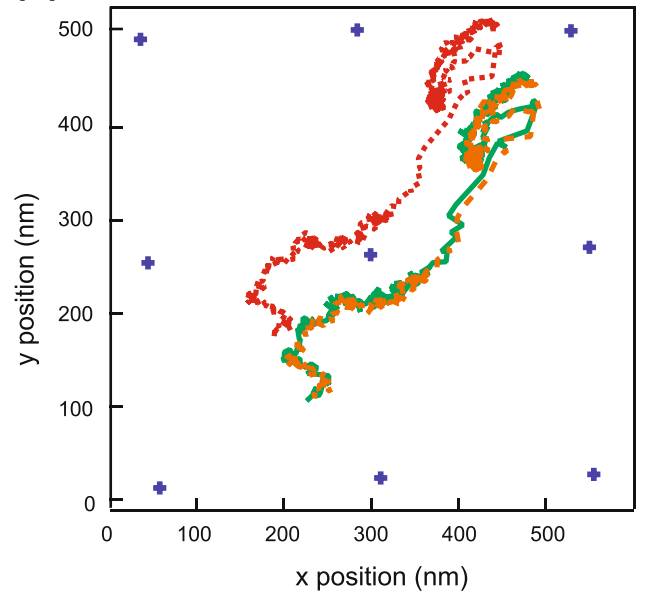

(b)

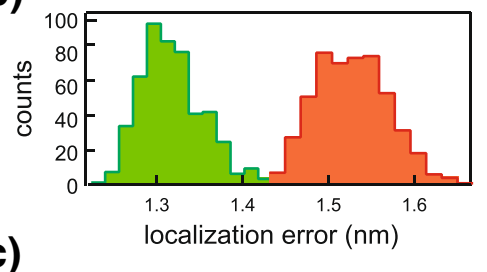

(c)

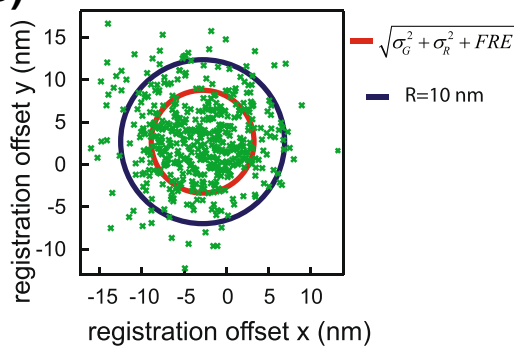

Figure 2 (a) $x$-y Trajectory of a $100 \mathrm{~nm}$ diameter fluorescent bead immobilized on the coverslip and imaged during a time lapse movie. The $x$ and $y$ displacement is due to the sample drift. Z position is stabilized by the axial feedback mechanism. Green trace is made of the measured position of the bead as seen in the green imaging channel. Red trace corresponds to the red imaging channel. The orange dashed trace is obtained after application of the registration function to the red points. Black crosses represent the positions of the fiducial markers. (b) Histograms of the localization precision after Gaussian fitting of the bead (weighted least squares). $1.5 \mathrm{~nm}$ and $1.3 \mathrm{~nm}$. (c) Scatter plot of the residual $x$ and $y$ offset $x_{g}-x_{r}$ and $y_{g}-y_{r}$. The blue circle has a radius of $10 \mathrm{~nm}$, the red circle has a radius given by $\sqrt{\sigma_{r}^{2}+\sigma_{g}{ }^{2}+F R E}$.

our study to the performance of three pairs of PAFPs. We investigated the pairs mEos2-Dronpa, mEos2- PSCFP2 and PSCFP2-PAMCherry1. The first two were originally discussed by Shroff et al. (Shroff et al. 2007), whereas the third one is an analogue of the PAMCherry1-PAGFP pair proposed by Lippincott-Schwartz and co-workers (Subach et al. 2009), as an alternative for PAGFP with PSCFP2. Although PAGFP is the archetype of photoactivatable probes, it has a relatively poor contrast ratio of 100 as compared to 1000 of PSCFP (Chudakov et al. 2007). Additionally, PSCFP2 can be visualized before the photoswitching allowing its localization in the sample whereas PAGFP is dark in its basal state.

\section{Fusion constructs}

We tested the performance of each pair by the use of chimaeric fusion constructs; surprisingly only a recent publication, that appeared during the review of the current manuscript (Renz et al. 2012) has detailed similar approach in cells. Engineering fusion pairs in principle provides two advantages: the exact same pattern is observed in each channel and the stoichiometry of the complex is fixed, allowing quantitative insight on the relative photoconversion and localization efficiency of the members of each pair. Purification of the constructs (as described in the Methods) allows performing both ensemble absorbance measurements as well as single molecule colocalization experiments. It is worthwhile to note that the latter provides also a prompt test of the registration accuracy of the setup. Although single molecule asynchronous colocalization experiments are beyond the scope of PALM microscopy, they can nevertheless be exploited towards a quantitative measurement of the number of labeled proteins present in a sample, an appealing development of PALM based imaging (Annibale et al. 2011a, b). Purified PAFPs were used in absorbance experiments in a cuvette using a spectrophotometer (Cary50, Varian) or immobilized in a PolyAcrylamide Matrix (15\% Acrylamide concentration, 1:18 crosslinker ratio) sandwiched between two clean glass coverslips. Experiment in cells were performed by fusing the double fluorophore chimeras to the plasma membrane shuttling short sequence SrcN15 (Annibale et al. 2011a). In most of the cases we investigated plasma membrane patches, obtained according to a previously published protocol (Perez et al. 2006) in order to minimize background autofluorescence.

\section{mEos2-Dronpa}

The first pair of photoactivatable fluorescent proteins proposed for dual color experiments was tdEos-Dronpa (Shroff et al. 2007). This combination evolved in mEos2Dronpa and it is in principle suitable for a sequential dual color imaging scheme, since the nominal reversible photoswitching ability of Dronpa allows to turn all of the fluorophore off and then to sparsely re-activate it again once the photoconversion of mEos2 is completed.

Throughout our experiments we have encountered two significant issues limiting this approach. From the studies of absorbance in solution of the two individual fluorophores, we observed that: first, the photoconversion of mEos2 in our hands was never complete, and second that the reversible activation of Dronpa in multiple cycles 
is limited, de facto, to a few switching cycles due to significant photobleaching. The evolution of the mEos 2 absorption spectrum at $\mathrm{pH}$ 8.5, upon $405 \mathrm{~nm}$ irradiation with a Xenon arc lamp (Lambda LS Xenon Arc Lamp System, Sutter Instrument) displayed in Additional file 1: Figure S4 a, indicates that the photoconversion follows a first order kinetics until saturation occurs, leaving a residual absorbance at $495 \mathrm{~nm}$, with a green to red concentration ratio of 1.4 indicative of a $60 \%$ photoconversion efficiency. Irradiation beyond this point results in a shift of the $510 \mathrm{~nm}$ isosbestic point, and an increase of the $270 \mathrm{~nm}$ band, hinting at a degradation of the chromophore. The single molecule in vitro colocalization experiment of purified mEos2-Dronpa fusion pair is shown in Figure 3a, and yields a $13 \%$ colocalization provided that the sample is sufficiently diluted and that the acquisition time of mEos 2 is short enough to minimize Dronpa bleaching (Table 1).

\section{PSCFP2-mEos2}

An immediate alternative is the PSCFP2-mEos2 pair, where instead of relying on the reversible photoswitching of the green protein of the pair, the much lower photoconversion efficiency of PSCFP2 can be exploited in order to achieve sequential imaging. PSCFP2(G) has an extinction coefficient similar to mEos2(G) (83\%), but a much lower Q.Y. $=0.2$, i.e. almost one quarter of mEos2(G). When the acquisition time moves above a value in the order of tens of minutes, much less PSCFP2 molecules are localized than mEos 2 , as displayed by the single molecule imaging of the fusion construct displayed in Figure 3b. Here we observe poor colocalization that is due to the photobleaching of PSCFP2 (C). Steady state absorbance (Additional file 1: Figure S4 c) of the purified fusion construct reveals that the efficiency in the formation of the chromophore is in favor of PSCFP2, with a 1:0.7 ratio to mEos2. Upon saturation of the activated form of mEos2, only a fraction of PSCFP2 has been photoconverted. After bleaching of the residual green fluorophores, photoconversion of PSCFP2 can be carried out until the number of localized single molecules saturates. PALM experiments in vitro showed a negligible colocalization fraction (Figure $3 \mathrm{~b}$, therefore confirming the observation arising from absorbance measurements that only a fraction of both species undergoes photoconversion and localization. Although this pair presents interesting perspectives, since the inactive forms of both proteins are fluorescent and can be exploited for other fluorescent techniques prior to photoconversion, the imbalance in the photoconversion ratios reflected by the negligible colocalization performance, prompted the search for a third pair.

\section{PSCFP2-PAMCherry 1}

Since the photophysics of mEos2 proves a limiting factor towards a truly parallel acquisition, the next pair we investigated PAMCherry1 as a possible replacement for mEos2 as a red partner in dual color PALM measurements. PAMCherry1 (Subach et al. 2009), developed by Lippincott-Schwartz and coworkers, is a photoactivatable

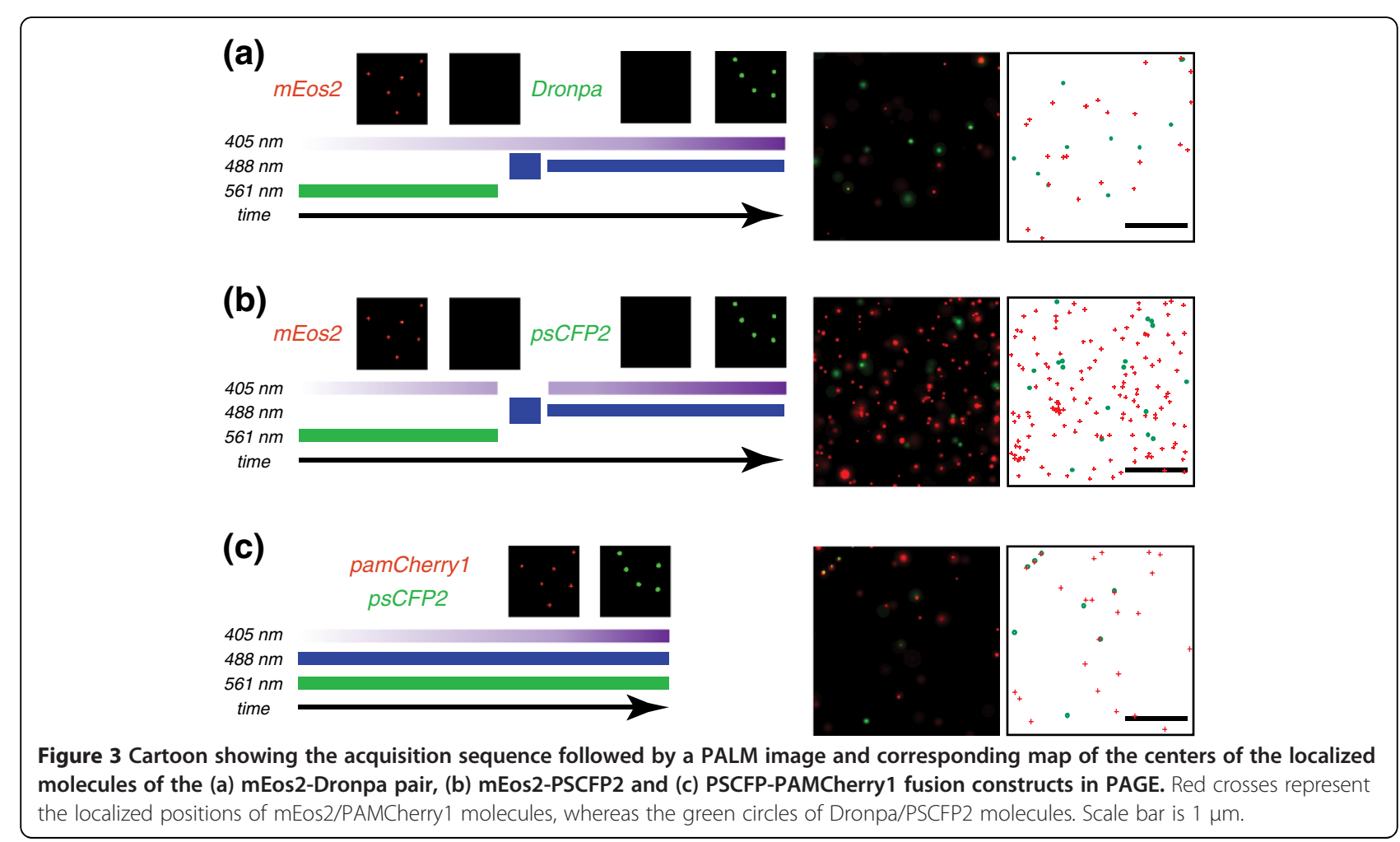


Table 1 Photoconversion efficiencies and colocalization fractions for the three fusion constructs investigated in this work

\begin{tabular}{llll}
\hline Fused fluorophore pair & First fluorophore efficiency & Second fluorophore efficiency & Colocalization fraction (\% of minority species) \\
\hline Dronpa-mEos2 & n.d. & $53 \%$ & $13 \%$ \\
\hline PSCFP2-mEos2 & $23 \%$ & $53 \%$ & $0 \%$ \\
\hline PSCFP2-PAMCherry1 & $28 \%$ & $58 \%$ & $15 \%$ \\
\hline
\end{tabular}

Colocalization fraction is defined as the number of fluorophore pairs falling within a $200 \mathrm{~nm}$ radius in a diluted sample. For constructs with mEos 2 colocalization fraction will depend on the number of localized mEos 2 molecules.

fluorescent protein undergoing irreversible transition from a non emissive state to a red form upon the usual $405 \mathrm{~nm}$ irradiation. The obvious advantage of a non emissive off state (as opposed to the green fluorescence of the mEos2 off -state) is that there will be no overlap at any time between the inactive form of PAMCherry1, and the active form of PSCFP2.

Investigation of the fusion construct in solution showed that, under the assumption of an initial 1:1 stoichiometry, the photoconversion efficiency of the two species is quite different. Approximately 58\% of the PAMCherry 1 molecules photoconverts effectively, whereas PSCFP2 photoconversion efficiency reaches $28 \%$ (Additional file 1 : Figure S4 d and Table 1). In the single molecule colocalization experiments reported in Figure 3c, the colocalization ratio is around $15 \%$, consistent with the $0.58 \times 0.28$ product expected from the photoconversion efficiencies measured by absorbance in solution. It should be also noted that PAMCherry1 photoactivation is elicited also by $488 \mathrm{~nm}$ light. Therefore, the excitation wavelength for PSCFP2 should be brought on in steps, allowing for a sparse photoactivation of the PAMCherry1 molecules. This colocalization value (15\%, see Table 1) is concentration independent, given the parallel acquisition of the two species. This is an important difference with respect to the previous two pairs, since the amount of localized PSCFP2 or Dronpa will depend on the number of mEos 2 molecules that is necessary to localize first.

\section{Asynchronous single molecule co-localization in PAGE}

The experiments performed in PAGE allow for a full control of the sample dilution. It is then possible to operate in a condition far from the super-resolution regime, e.g. where approximately one molecule per $\mu \mathrm{m}^{2}$ is present, and therefore all pairs of fluorophores of different color falling within a distance of, e.g. $200 \mathrm{~nm}$ one from the other can be considered with high likelihood as belonging to the same pair. As previously mentioned, the distance between these two pairs may deviate from the root of the squared localization errors for multiple reasons, prominently the drift of the setup between the instant of localization of two adjacent molecules.

We shall again remember that this type of experiment is a form of asynchronous colocalization, since the time interval between the localization of two members of a heterodimer is stochastic and greater than zero. Figure 4, displays the distances between the proteins that form PSCFP2-PAMCherry1 fusion pair as a function of the temporal separation of their localization instant. Two populations are visible, one around $200 \mathrm{~nm}$ separation, the other one where the particles are separated below $50 \mathrm{~nm}$. The measured distances are in almost all cases larger than their errors (root of the squared sum of the localization precisions in each channel), confirming that the asynchronous nature of the measurement as well as the registration accuracy introduce an additional error to the measured co-localization distance besides the localization precision. We attribute the population with the larger deviation to molecules suffering a residual diffusion within the pores of the PolyAcrylamide gel. On the other hand, the group of molecules localizing within $50 \mathrm{~nm}$ provides us with two important informations: first, there is no significant drift of the colocalization as a function of time, indicating that the overall stabilization of the setup is effective. Second, the fact that the co-localization distances of the second group have an average value of $\sim 30 \mathrm{~nm}$ indicates that the setup is operating close to its registration limit $\left(\sqrt{\sigma_{r}^{2}+\sigma_{g}^{2}+e R E G^{2}}\right)$.

\section{Addressing molecular photophysics}

As we have extensively discussed in two previous publications (Annibale et al. 2011a, b), identification of molecules

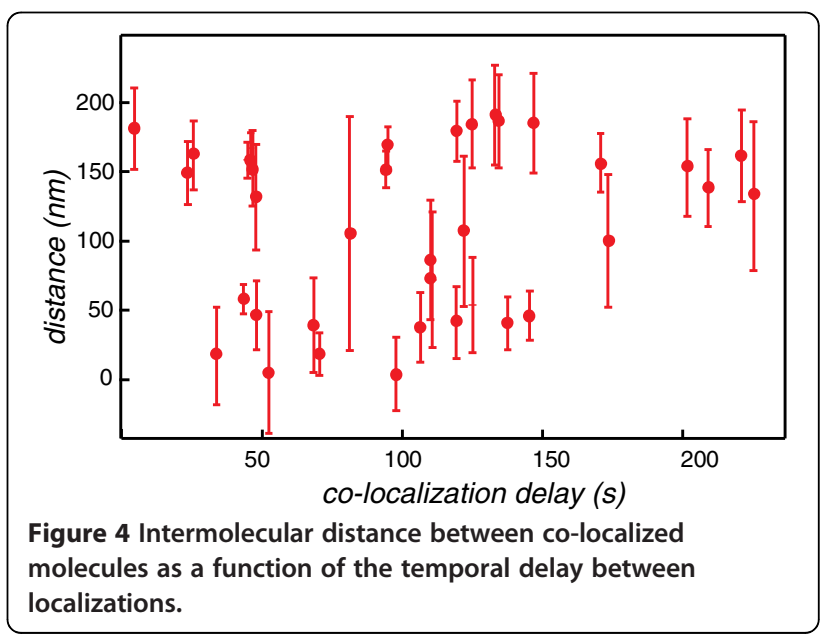


should carefully take into account the presence of molecular reactivations, that may lead to photophysical artifacts. The stochastic condition for photoactivation at the basis of PALM microscopy implies that in any given point the number of activated molecule per unit time obeys a Poisson's flow, and depends only on the local density (molecules/ $\mu \mathrm{m}^{2}$ ) and on the photoactivation rate (new molecules/s).

Once a spatial cluster is identified by means of density thresholding, the known observables are the number of molecules $n_{c}$ within the cluster, the time elapsed between the first and last localization $\tau_{\mathrm{c}}$ and the total duration of the PALM acquisition $T$.

Stochastic activation implies that the activation and localization of the molecules in the cluster should be homogeneously distributed through the entire duration of the acquisition $\mathrm{T}$.

The ratio between the number of localized molecules and the experiment duration determines the steady-state photo-activation rate $r$. The product $r^{*} T_{c}$ determines the average number of molecules $m$ expected to be localized in this time interval. $m$ is the average of a Poisson's distribution, that can be used to assess the probability $p$ of detecting $n_{c}$ molecules within the cluster. This probability is then used to score each cluster.

An example, applied to an experimental dataset containing a mixture of mEos2 and Dronpa molecule imaged in vitro is displayed in Additional file 1: Figure S5. Clusters with a number of molecules $n_{c}$ and a duration $\tau_{c}$ such that $p$ is less that 0.01 are rejected as temporal artifacts and they are replaced by a localization event having a $(x, y)$ position calculated based on the weighted mean (using the localization precision $s$ as a weight) of the position of all the $n_{c}$ molecules and a localization precision given by their standard deviation.

\section{Application}

After the identification of best performing protein pair, viz. PSCFP2-PAMCherry1 together with the discussed methods we have chosen to test our approach on a biological process of the $\beta 2$ adrenergic receptor ( $\beta 2-\mathrm{AR})$, a prototypical G Protein-Coupled Receptor (GPCRs), i.e. agonist stimulated receptor endocytosis. The formation of clathrin coated pits represents the main pathway for receptor mediated endocytosis, (for a recent review on the subject, see (Marchese et al. 2008)) mainly upon stimulation regulated by an external agonist. Signaling receptors such as GPCRs are one important cargo of clathrin coated vesicles, and they tend to internalize rapidly after stimulation and interaction with the adaptor proteins $\beta$-arrestins. The sub-diffraction limit feature size of forming GPCR endosomes and aggregates of segregated receptor on the plasma membrane are an appealing system to be studies using super-resolution techniques (Scarselli et al. 2012). $\beta 2$-AR was labeled using PSCFP2, whereas PAMCherry1 fused to clathrin light chain was used. For each species, after the clustering threshold, the information about two subgroups of molecules is available: on the one hand those molecules that are part of clusters, and on the other hand all the molecules (including the centers of mass of temporal clusters) that compose the so called monomer fraction. The first group can either be displayed alone to provide a selective image of only the physical clusters present in the sample, a strategy already employed for STORM data (Jones et al. 2011), or merged with the monomer fraction in order to obtain an artifact free PALM image.

Figure 5 displays the dual color TIRF and PALM images of a region of the plasma membrane of a HeLa cell expressing $\beta 2$-AR and clathrin after stimulation with the agonist isoproterenol. Panel a displays a DIC image together with the overlay of the region imaged in PALM. Small puncta corresponding to the clathrin coated vesicles are visible already at this resolution level. Panels b and $c$ display respectively the red and green TIRF images of the region within the square in a, and panel c displays the overlay of the two channel TIRF images. A large amount of aggregates in both imaging channel is visible, with some evident colocalization events. Panel d displays the corresponding dual color raw PALM image. A much clearer picture emerges from the PALM image displaying only the clustered fraction and the corresponding map of the localized fluorescence centers (panels e and f). In this representative figure, more than ten clathrin coated vesicles are detectable, and approximately five of them are clearly associated to $\beta 2$-AR clusters. An inset showing one of these colocalization events is displayed in panels g (TIRF) and $\mathrm{h}$ (PALM). The amount of co-clustering observed is consistent with the observation from a positive control.

We performed the additional positive control experiment by co-transfecting HeLa cells using clathrin light chain (clc) fusions with PSCFP2 and pamCherry1 (data not shown). By using the approach described in the ms, we observed in total 158 clathrin clusters in the red channel, 240 in the green channel and in 70 cases we could observe an overlap.

\section{Effect of chemical fixation}

The final point to be discussed in the framework of super-resolution co-localization is the assumption that the cell under observation can be frozen in a definite configuration by means of chemical fixation. The most commonly used fixatives in cell biology are aldehydes, most notably formaldehyde and glutaraldehyde. Typically, the slower acting formaldehyde is complemented by the addition of glutaraldehyde, to speed up the reaction and achieve a better fixation, in particular of membrane proteins. It has been demonstrated that unless glutaraldehyde is used, a significant fraction of both 

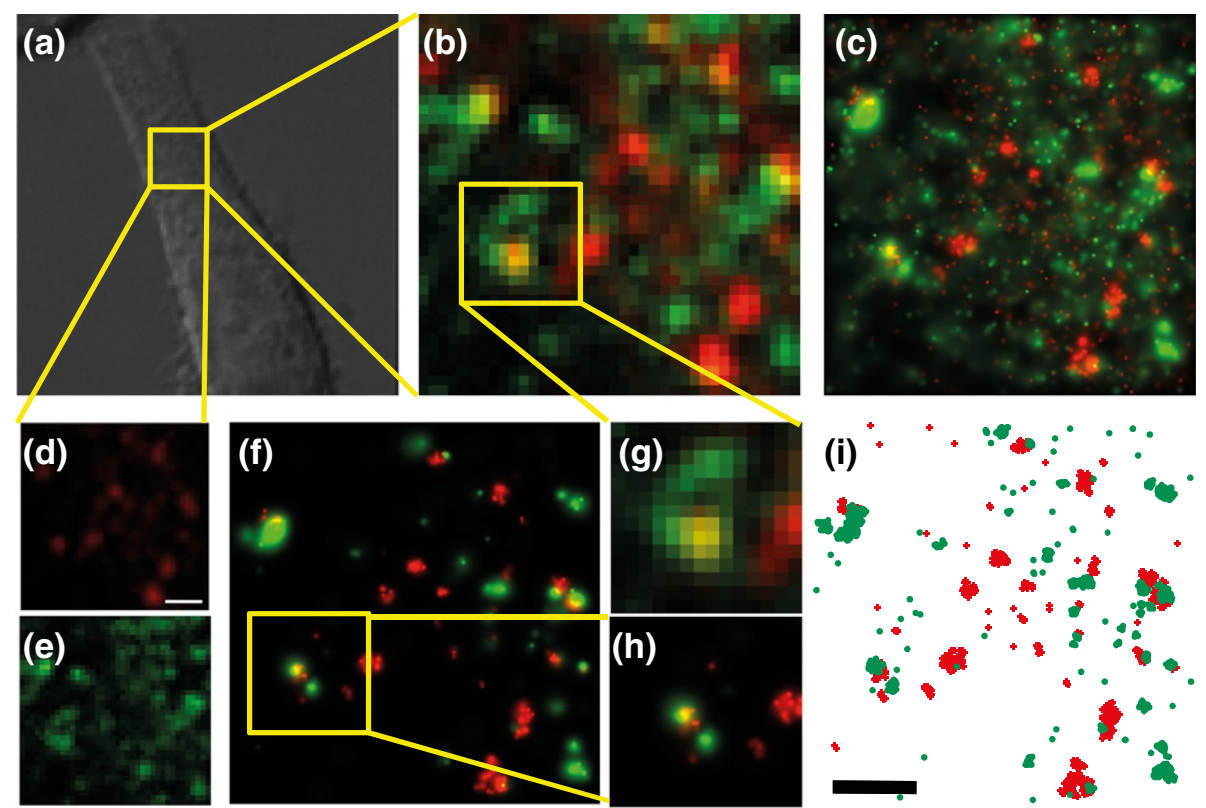

Figure 5 (a) DIC image of HeLa cell and overlay of red channel PALM image showing clathrin coated pits on the plasma membrane. (b) and (c) TIRF green and red channels of the selected region in (a) (d) dual color TIRF image of the region selected in a. (e) raw dual color PALM image of the region corresponding to (d). (f) Dual color PALM image of the clustered fraction only ( $\mathbf{g}$ ) and (h) show a detail of a colocalization event between a clathrin coated pit and a $\beta 2-A R$ cluster in TIRF and PALM (clustered fraction) respectively (i) localized map of the position of each fluorophore in both channel corresponding to $(\mathbf{f})$. Scale bar is $1 \mu \mathrm{m}$.

membrane anchored or transmembrane proteins remains mobile (Tanaka et al. 2010).

Additional file 1: Figure S6 displays the mobile fraction at different lag times, for the three sub-sets of our dataset. The mobile component prior to fixation and calculated using the method developed by Schutz et al. (Schutz et al. 1997) amounts to $75 \%$ of the total traces, decreasing to $45 \%$ after an average of $30 \mathrm{~s}$, indicating that only $40 \%$ of the mobile molecules was immobilized after $15 \mathrm{~s}$ incubation with the fixative mix. Considering that, given the typical diffusion coefficient of a transmembrane protein is in the range of $0.01-1 \mu \mathrm{m}^{2} / \mathrm{s}$ and the MSD scales as $\left\langle r^{2}\right\rangle=4 D t$, after $15 \mathrm{~s}$ we are in the upper range of $6 \mu \mathrm{m}^{2}$. After an average of $90 \mathrm{~s}$, and an upper bound for the mean square displacement of $18 \mu \mathrm{m}^{2}, 33 \%$ of the originally mobile molecules are still diffusing.

A small cluster, such as the clathrin coated vesicle at the plasma membrane, or a small receptor aggregate resulting from the temporary response to a stimulus, would be clearly blurred out or even dissolved if it contains a few molecules, before complete fixation has occurred. A residual diffusion after $1.5 \mathrm{~h}$ from fixation was observed also for clathrin (data not shown). Significantly, the immobile fraction remained at around 30\% before and after fixation, but after fixation the mobile clathrin molecules appeared to be confined to a range $\left(\mathrm{MSD}^{0.5}\right)$ of $\sim 0.275 \mu \mathrm{m}$.

\section{Conclusions}

In this paper, we investigated and critically reviewed the key technical steps and associated challenges encountered in multicolor super-resolution localization microscopy. We proposed an active stabilization mechanism based on a totally internally reflected laser line to compensate the effect of axial drift, to enhance long term stability (nm-level) and to take full advantage of the registration accuracy of the setup.

We identified the limit to the registration accuracy of our stabilized widefield setup over time, finding an effective registration precision systematically larger than the FRE, the typical benchmark used in these experiments. Then, we reviewed the original dualcolor PALM pair Dronpa-mEos2, the more recent mEos2-PSCFP2 and finally we settled on the pair PAMCherry1-PSCFP2, reporting an application regarding the clustering of the $\beta 2$-AR during clathrin-dependent endocytosis, providing also a quantitative method for the rejection of those clusters that clearly arise due to photophysics.

We concluded this paper with the observation that the requirements for long term stability do not apply only to the setup, but also to the sample itself. We investigated the diffusional properties of the $\beta 2$-AR after fixation, observing that the immobilization of membrane bound proteins is a slow process, leaving a significant fraction of mobile molecules diffusing for up to minutes after fixation. 
The conclusion that can be drawn from these experiments highlight the significant challenges that PALM and similar techniques face in relation to dual-color imaging if they want to stand up to the definition of super-resolution methods also in multicolor mode. The origin of certain limitations, such as the registration precision larger than $10 \mathrm{~nm}$, is not obvious. Registration accuracies below $1 \mathrm{~nm}$ (Pertsinidis et al. 2010) were demonstrated only in particular conditions, in vitro and over limited field of views (one pixel). The use of fusion constructs to identify the best candidate among the possible combination of PAFPs is also an approach that yielded interesting information about the final stoichiometries that can be achieved with these constructs. The couple PAMCherry1-PSCFP2 is the only pair that does not require sequential imaging, maximizing the speed and at the same time increasing the quantitative outcome of the molecular counting process. The combination of a $>10 \mathrm{~nm}$ registration precision, $>20 \mathrm{~nm}$ localization precision and $60 \%$ photoconversion efficiency in the best case effectively rules out the use of PALM to probe heter-oligomerization processes quantitatively. However, investigation of co-clustering of larger and denser structures remains a promising avenue.

\section{Additional file}

Additional file 1: Figure S1. Registration protocol. (a) Dual-color image of the grid obtained by integrating the images of a fiducial fluorescent bead scanned across a square matrix of approximately $10 \mu \mathrm{m}$ side. (b) Average localization precision of the individual fiducial nanoparticles as a function of the number of integrated frames (equivalent to multiples of the exposure time). (c) Average Fiducial Registration Error following the Local Weighted Mean mapping of all the coordinates of the fiducial marker in the red channel and subtraction from the corresponding coordinates in the green. Figure S2. Schematic of the protocol employed in a dual-color imaging experiment applicable to any laboratory or commercial localization microscopy measurement. Figure S3. Measurement of the lateral drift. Upper graph: absolute drift, in the red and green channel, of one fiducial nanoparticle in a mEos2-PSCFP2 sample. Bottom graph: relative drift in the $\mathrm{x}$ and $\mathrm{y}$ directions. Lower inset: scatter plot of the residual offset between the green and red channel after approximately $520 \mathrm{~s}$ from the beginning of the acquisition till the end. The red circle has a radius of $10 \mathrm{~nm}$. Figure S4. (a) Evolution of the absorption spectrum of mEos 2 upon $405 \mathrm{~nm}$ irradiation, at pH 7.9. It is important to notice that the photoconversion of the red form reaches a saturation before that the green form ( $488 \mathrm{~nm}$ band) has completely disappeared. The ratio between the concentration of the green species and the red one at saturation is approximately 1.8. (b) mEos2 bands before and after photoconversion ( $1 \mathrm{~h}$ ) in a $4-15 \%$ gradient denaturing Blue Coomassie gel. (c) Absorption spectrum of PSCFP2-mEos2 fusion (after detrending to remove an exponential background). The evolution of the absorption spectrum is displayed in a color code up to 75 minutes of $405 \mathrm{~nm}$ irradiation, when no further increase of the absorbance of the mEos2(R) form is observed. The dashed line represents the theoretical PSCFP2-mEos2 spectrum (prior to photoconversion) at the expected 1:1 stoichiometry, normalized in order to match the peak mEos2(G) absorbance. An excess (1.3x) of PSCFP2 is observed. (d) Ensemble spectroscopic characterization of PSCFP2-PAMCherry1 fusion protein. Evolution of the absorption spectrum upon $405 \mathrm{~nm}$ laser light irradiation. Extinction coefficients for each species are quoted, and allow to reconstruct the final concentration of activated species.

Figure 55. (a) Map of the localized centers for mEos2 (red) and Dronpa (green) molecules activated in PAGE at low concentration. (b) Two arbitrary clusters are selected and their kymographs displayed. (c) and (d). (e) The number of molecules activated in time for each species is displayed. Linear guide for the eye outlines the linear activation regime where the assumption of a Poisson flow for the arrival time of the localization events hold. (f) Cumulative Distribution Functions for a Poisson Distribution calculated for the two clusters displayed in b. $m_{-m E o s}=9$ and $m_{-D r o n p a}=23$. Figure S6. (a) $\beta 2$-mEos2 traces before fixation on the plasma membrane of a HeLa cell. (b) After an average of $30 \mathrm{~s}$ after fixation. (c) After an average of $90 \mathrm{~s}$ after fixation. Scale bar $5 \mu \mathrm{m}$. (d) Evolution of the mobile fraction of $\beta 2$-ARs on the plasma membrane of a HeLa cell as a function of the time from fixation.

\section{Competing interests}

The authors declare that they have no competing interests.

\section{Authors' contributions}

PA, MG and MS performed the measurements presented in the paper and supplementary material. PA built PALM setup and analyzed data presented in the paper and supplementary material. MG wrote the registration code. MS built initial constructs and validated them in vitro and in vivo. PA conceived the study with AR and prepared the manuscript. All the authors read and approved the final manuscript.

\section{Acknowledgements}

This work was financially supported by FNS grants no. 200021-125319 and no 20021-132206. We would like to acknowledge Mike Davidson for providing PAMCherry-clathrin light chain and PSCFP2-clathrin light chain, Lely Feletti for professional assistance in sample preparation as well as Annalisa Ansaloni. We would like to acknowledge insightful suggestions regarding the purification strategy of mEos2 from Prof. Hilal Lashuel and Dr. Ranieri Bizzarri. We would also like to thank Prof. Melike Lakadamyali for useful discussion.

\section{Author details}

'Laboratory of Nanoscale Biology, Institute of Bioengineering, School of Engineering, EPFL, Lausanne 1015, Switzerland. Present address: Department of Neuroscience, University of Pisa, Pisa, Italy.

Received: 5 September 2012 Accepted: 21 November 2012 Published: 24 November 2012

\section{References}

Andresen M, Stiel AC, Folling J, Wenzel D, Schonle A, Egner A, Eggeling C, Hell SW, Jakobs S (2008) Photoswitchable fluorescent proteins enable monochromatic multilabel imaging and dual color fluorescence nanoscopy. Nat Biotechnol 26:1035-1040

Annibale P, Vanni S, Scarselli M, Rothlisberger U, Radenovic A (2011a) Identification of clustering artifacts in photoactivated localization microscopy. Nat Methods 8:527-528

Annibale P, Vanni S, Scarselli M, Rothlisberger U, Radenovic A (2011b) Quantitative Photo Activated Localization Microscopy: Unraveling the Effects of Photoblinking. PLoS One 6(7):e22678

Baddeley D, Crossman D, Rossberger S, Cheyne JE, Montgomery JM, Jayasinghe ID, Cremer C, Cannell MB, Soeller C (2011) 4D super-resolution microscopy with conventional fluorophores and single wavelength excitation in optically thick cells and tissues. PLoS One 6:e20645

Bates M, Dempsey GT, Chen KH, Zhuang XW (2012) Multicolor Super-Resolution Fluorescence Imaging via Multi-Parameter Fluorophore Detection. Chemphyschem 13:99-107

Bates M, Huang B, Dempsey GT, Zhuang X (2007) Multicolor super-resolution imaging with photo-switchable fluorescent probes. Science 317:1749-1753

Betzig E, Patterson GH, Sougrat R, Lindwasser OW, Olenych S, Bonifacino JS, Davidson MW, Lippincott-Schwartz J, Hess HF (2006) Imaging intracellular fluorescent proteins at nanometer resolution. Science 313:1642-1645

Buckers J, Wildanger D, Vicidomini G, Kastrup L, Hell SW (2011) Simultaneous multi-lifetime multi-color STED imaging for colocalization analyses. Opt Express 19:3130-3143

Chichili GR, Rodgers W (2007) Clustering of membrane raft proteins by the actin cytoskeleton. J Biol Chem 282:36682-36691 
Chudakov DM, Lukyanov S, Lukyanov KA (2007) Tracking intracellular protein movements using photoswitchable fluorescent proteins PS-CFP2 and Dendra2. Nat Protoc 2:2024-2032

Churchman LS, Okten Z, Rock RS, Dawson JF, Spudich JA (2005) Single molecule high-resolution colocalization of Cy3 and Cy5 attached to macromolecules measures intramolecular distances through time. Proc Natl Acad Sci USA 102:1419-1423

Dempsey GT, Vaughan JC, Chen KH, Bates M, Zhuang XW (2011) Evaluation of fluorophores for optimal performance in localization-based super-resolution imaging. Nat Methods 8:1027

Engelhardt J, Keller J, Hoyer P, Reuss M, Staudt T, Hell SW (2011) Molecular Orientation Affects Localization Accuracy in Superresolution Far-Field Fluorescence Microscopy. Nano Lett 11:209-213

Frost NA, Shroff H, Kong H, Betzig E, Blanpied TA (2010) Single-molecule discrimination of discrete perisynaptic and distributed sites of actin filament assembly within dendritic spines. Neuron 67:86-99

Goshtasby A (1988) Registration of Images with Geometric Distortions. leee T Geosci Remote 26:60-64

Hess ST, Girirajan TPK, Mason MD (2006) Ultra-high resolution imaging by fluorescence photoactivation localization microscopy. Biophys J 91:4258-4272

Hsu CJ, Baumgart T (2011) Spatial association of signaling proteins and F-actin effects on cluster assembly analyzed via photoactivation localization microscopy in T cells. PLoS One 6:e23586

Huang B, Jones SA, Brandenburg B, Zhuang XW (2008) Whole-cell 3D STORM reveals interactions between cellular structures with nanometer-scale resolution. Nat Methods 5:1047-1052

Jones SA, Shim SH, He J, Zhuang XW (2011) Fast, three-dimensional superresolution imaging of live cells. Nat Methods 8:499-U496

Lehmann M, Rocha S, Mangeat B, Blanchet F, Uji IH, Hofkens J, Piguet V (2011) Quantitative multicolor super-resolution microscopy reveals tetherin HIV-1 interaction. PLoS Pathog 7:e1002456

Manders EMM, Stap J, Brakenhoff GJ, Vandriel R, Aten JA (1992) Dynamics of 3Dimensional Replication Patterns during the S-Phase, Analyzed by Double Labeling of DNA and Confocal Microscopy. J Cell Sci 103:857-862

Manders EMM, Verbeek FJ, Aten JA (1993) Measurement of Colocalization of Objects in Dual-Color Confocal Images. J Microsc-Oxford 169:375-382

Marchese A, Paing MM, Temple BRS, Trejo J (2008) G protein-coupled receptor sorting to endosomes and lysosomes. Annu Rev Pharmacol Toxicol 48:601-629

Mortensen Kl, Churchman LS, Spudich JA, Flyvbjerg H (2010) Optimized localization analysis for single-molecule tracking and super-resolution microscopy. Nat Methods 7:377-U359

Nugent-Glandorf L, Perkins TT (2004) Measuring 0.1-nm motion in $1 \mathrm{~ms}$ in an optical microscope with differential back-focal-plane detection. Opt Lett 29:2611-2613

Patterson G, Davidson M, Manley S, Lippincott-Schwartz J (2010) Superresolution imaging using single-molecule localization. Annu Rev Phys Chem 61:345-367

Perez JB, Martinez KL, Segura JM, Vogel H (2006) Supported cell-membrane sheets for functional fluorescence imaging of membrane proteins. Adv Funct Mater 16:306-312

Pertsinidis A, Zhang YX, Chu S (2010) Subnanometre single-molecule localization, registration and distance measurements. Nature 466:647-U611

Renz M, Danielbs BR, Vamosi G, Arias IM, Lippincott-Schwartz J (2012) Plasticity of the asialoglycoprotein receptor deciphered by ensemble FRET imaging and single-molecule counting PALM imaging. Proc Natl Acad Sci USA 109(44): E2989-E2997

Rust MJ, Bates M, Zhuang XW (2006) Sub-diffraction-limit imaging by stochastic optical reconstruction microscopy (STORM). Nat Methods 3:793-795

Scarselli M, Annibale P, Radenovic A (2012) Cell Type-specific beta 2-Adrenergic Receptor Clusters Identified Using Photoactivated Localization Microscopy Are Not Lipid Raft Related, but Depend on Actin Cytoskeleton Integrity. J Biol Chem 287:16768-16780

Schutz GJ, Schindler H, Schmidt T (1997) Single-molecule microscopy on model membranes reveals anomalous diffusion. Biophys J 73:1073-1080

Shroff H, Galbraith CG, Galbraith JA, White H, Gillette J, Olenych S, Davidson MW, Betzig E (2007) Dual-color superresolution imaging of genetically expressed probes within individual adhesion complexes. Proc Natl Acad Sci USA 104:20308-20313

Subach FV, Patterson GH, Manley S, Gillette JM, Lippincott-Schwartz J, Verkhusha W (2009) Photoactivatable mCherry for high-resolution two-color fluorescence microscopy. Nat Methods 6:153-159
Subach FV, Patterson GH, Renz M, Lippincott-Schwartz J, Verkhusha W (2010) Bright monomeric photoactivatable red fluorescent protein for two-color super-resolution sptPALM of live cells. J Am Chem Soc 132:6481-6491

Tanaka KAK, Suzuki KGN, Shirai YM, Shibutani ST, Miyahara MSH, Tsuboi H, Yahara M, Yoshimura A, Mayor S, Fujiwara TK, Kusumi A (2010) Membrane molecules mobile even after chemical fixation. Nat Methods 7:865-866

Testa I, Wurm CA, Medda R, Rothermel E, von Middendorf C, Folling J, Jakobs S, Schonle A, Hell SW, Eggeling C (2010) Multicolor fluorescence nanoscopy in fixed and living cells by exciting conventional fluorophores with a single wavelength. Biophys J 99:2686-2694

Wilmes S, Staufenbiel M, Lisse D, Richter CP, Beutel O, Busch KB, Hess ST, Piehler J (2012) Triple-color super-resolution imaging of live cells: resolving submicroscopic receptor organization in the plasma membrane. Angew Chem Int Ed Engl 51:4868-4871

Yao J, Fetter RD, Hu P, Betzig E, Tjian R (2011) Subnuclear segregation of genes and core promoter factors in myogenesis. Genes Dev 25:569-580

doi:10.1186/2192-2853-1-9

Cite this article as: Annibale et al:: Identification of the factors affecting co-localization precision for quantitative multicolor localization microscopy. Optical Nanoscopy 2012 1:9.

\section{Submit your manuscript to a SpringerOpen ${ }^{\odot}$ journal and benefit from:}

- Convenient online submission

- Rigorous peer review

- Immediate publication on acceptance

- Open access: articles freely available online

- High visibility within the field

- Retaining the copyright to your article

Submit your next manuscript at $>$ springeropen.com 